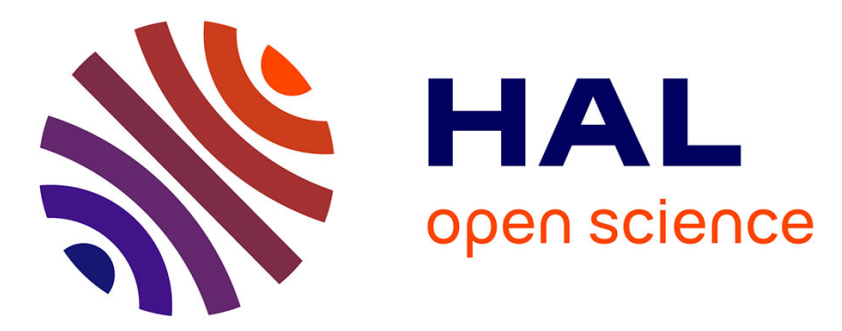

\title{
Pretransitional effects in isotropic phases close to nematic, nematic discoid and columnar phases
}

Cédric Baumann, J.P. Marcerou, J.C. Rouillon, Jacques Prost

\section{To cite this version:}

Cédric Baumann, J.P. Marcerou, J.C. Rouillon, Jacques Prost. Pretransitional effects in isotropic phases close to nematic, nematic discoid and columnar phases. Journal de Physique, 1984, 45 (3), pp.451-458. 10.1051/jphys:01984004503045100 . jpa-00209776

\section{HAL Id: jpa-00209776 https://hal.science/jpa-00209776}

Submitted on 1 Jan 1984

HAL is a multi-disciplinary open access archive for the deposit and dissemination of scientific research documents, whether they are published or not. The documents may come from teaching and research institutions in France or abroad, or from public or private research centers.
L'archive ouverte pluridisciplinaire HAL, est destinée au dépôt et à la diffusion de documents scientifiques de niveau recherche, publiés ou non, émanant des établissements d'enseignement et de recherche français ou étrangers, des laboratoires publics ou privés. 


\title{
Pretransitional effects in isotropic phases close to nematic, nematic discoid and columnar.phases
}

\author{
C. Baumann, J. P. Marcerou, J. C. Rouillon and J. Prost \\ Centre de Recherche Paul Pascal, Domaine Universitaire, 33405 Talence Cedex, France
}

(Reçu le 3 juin 1983, accepté le 21 novembre 1983)

\begin{abstract}
Résumé. - Nous présentons et discutons des résultats concernant les corrélations angulaires dans les phases isotropes des octyl et nonylcyanobiphényles, d'hexaalcanoates de truxène et d'hexaalcanoates de triphénylène. Pour les trois premiers composés, les corrélations sont dues à la proximité d'une phase nématique, alors que pour le dernier, elles sont liées à celle d'une phase colonne. Des écarts à la théorie de de Gennes sont mis en évidence et discutés dans une approximation de modes couplés.
\end{abstract}

\begin{abstract}
We report and discuss experimental results concerning pretransitional angular correlations in the isotropic phases of octyl and nonylcyanobiphenyls, truxene hexaalkanoate and triphenylene hexaalkanoate compounds. In the first three, the correlations are due to the proximity of a nematic phase, whereas in the last it is linked to the existence of a.columnar one Deviations from de Gennes theory are reported and discussed in a mode coupling approximation.
\end{abstract}

\section{Introduction.}

Flexoelectricity which was long thought to be characteristic of the liquid crystalline state, has been shown to exist in isotropic as well as [1] in anisotropic phases. This phenomenon is cooperative in the second case and due to anisotropic fluctuations in the first. A simple although not complete picture is obtained by taking account of electric quadrupole densities [2]. Indeed in any uniaxial (a fortiori biaxial) system, a macroscopic electric quadrupole exists, and couples to electric field gradients : this gives rise to flexoelectricity. In isotropic phases, fluctuating quadrupolar densities correspond to fluctuating anisotropic domains. They again couple to field gradients : the resulting birefringence is characteristic of flexoelectricity (linearity in the applied field) [3]. This provides an original probe to study pretransitional angular correlations.

The interdigital electrodes technique, used to detect this induced birefringence has been described previously [4]. Its use in the study of the isotropic phase of MBBA (1), showed that the information one can obtain, is essentially equivalent to that obtained with light scattering, Cotton-Mouton, Kerr effects, shear induced birefringence, etc. Apart from showing the existence of flexoelectricity, its main interest is to require only a very small amount of compound $(\sim 20 \mathrm{mg})$, which is useful in the study of newly synthesized compounds, and avoids turbidity cor- rections in the interpretation of experiments (optical path in the sample $\lesssim 100 \mu \mathrm{m})$. Another interesting feature is the frequency dynamic range (a few $\mathrm{Hz}$ to a few $\mathrm{MHz}$ ) which is well adapted to the measurement of orientational correlation times, close to transitions.

In section two we give a brief description of the experiment and a few elements necessary for its understanding.

The third section is devoted to the experimental results : the behaviour of the octyl and nonyloxycyanobiphenyls (8CB and $9 \mathrm{CB}$ ), truxene hexaalkanoate and triphenylene hexaalkanoate is investigated [5]. The first two compounds have been chosen as typical elongated mesogens; they present the advantage of being chemically stable; furthermore the nematic range decreases drastically from $C_{8}$ to $C_{9}$, which leads to the possibility of detecting the influence of the lower smectic phase. The second two are disk-like mesogens : the truxene derivative exhibits a nematic phase below the isotropic one [6] whereas the triphenylene reveals a columnar phase $\left(D_{\text {hd }}\right)[7]$.

In the fourth section we give a theoretical interpretation which takes account of the translation-orientation cross correlations. The mode coupling approach proposed here which generalizes the one given in [8], provides a satisfactory understanding of the pretransitional phenomena close to columnar and smectic A phases. 


\section{Experimental procedure.}

In order to study flexoelectricity in isotropic phases, one must first generate electric field gradients, and then detect the induced birefringence. As a matter of fact, such a program was proposed by Debye long ago [9] and achieved by Buckingham [10] somewhat later. The geometry they used, is however only suitable for the study of gases (and hence the determination of molecular quadrupoles). Interdigitated electrodes (Fig. 1), permit creation of a field periodic in one direction of space ( $\hat{x}$ axis of Fig. 1) and essentially exponentially decaying in a second one ( $\hat{z}$ axis of Fig. 1). At a given temporal frequency the fundamental Fourier component of the electric potential reads :

$$
\Phi(\mathbf{r}, t)=\Phi_{0} \mathrm{e}^{i q x} \mathrm{e}^{-q z} \mathrm{e}^{i \omega t}
$$

where $q=2 \Pi / \lambda_{\mathrm{c}}\left(\lambda_{\mathrm{c}}\right.$ period of the electric field $)$. $\Phi_{0} \simeq V / 3.7(V$ actual peak voltage applied to the electrodes).

Thus field gradients $\nabla_{x} E_{x}, \nabla_{x} E_{z}, \nabla_{z} E_{x}$ and $\nabla_{z} E_{z}$, all periodic along $x$ and exponentially decaying along $z$, are applied to any sample sitting on top of the interdigitated structure. Following de Gennes [11] one can describe orientational fluctuations by any symmetric traceless tensor $S_{i j}(\mathbf{r})$, the mean value of which is zero in isotropic phases (a good choice is for instance $S_{i j}=\Delta \mu^{-1}\left(\mu_{i j}-\frac{1}{3} \mu_{k k} \delta_{i j}\right)$ as explained in [12], in which $\mu_{i j}(\mathbf{r})$ is the local diamagnetic susceptibility and $\Delta \mu=\mu_{\|}-\mu_{\perp}$ is the magnetic anisotropy of a perfectly oriented sample).

From linear response theory one expects :

$$
S_{i j}=\chi \nabla_{i} E_{j}
$$

$\chi$ is the susceptibility characteristic of the nematic order. For a weakly first-order transition :

$$
\chi \propto\left(T-T_{\mathbf{N}}^{*}\right)^{-\gamma} .
$$

If mean field exponents hold, which seems to be correct in the pure isotropic-nematic case [12] $\gamma=1$ ( $T_{\mathrm{N}}^{*}$ is not too far from $T_{\mathrm{N}}$ the actual transition temperature).

Formula 2 shows that the geometry of the induced order is exactly that of the electric field gradient : it is biaxial as in shear flow induced birefringence, and periodic in the $x$ direction. As shown on figure $1 b$ the principal axes are mutually orthogonal. The period of the optical dielectric modulation is $\lambda_{e}$, as in the nematic phase, but the optical axis undergoes a full $\Pi$ rotation, unlike in the nematic phase (in which the induced mode is hydrodynamic; e.g. small deviation from the equilibrium direction). The corresponding phase grating will scatter light with the following characteristics :

- the wavevector conservation law allows selection of the wavelength $\lambda_{\mathrm{e}}$, corresponding to the linear electrooptic effect described above;

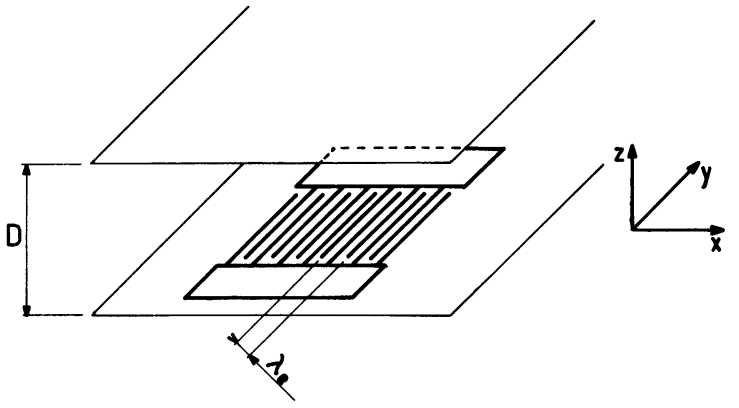

a)

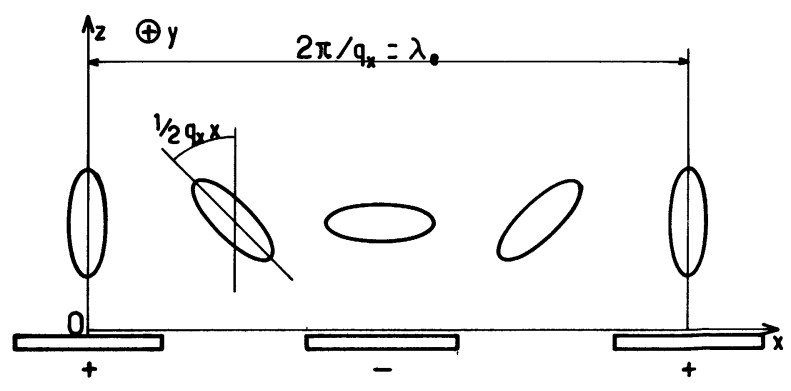

b)

Fig. 1. - a) Schematic representation of interdigitated electrodes, deposited on a glass slide. Note the definition of the field period $\dot{\lambda}_{\mathrm{e}}$ and the reference axes. $b$ ) The ellipse, and the $\hat{y}$, axes define the principal axes of the fundamental Fourier component of the induced (biaxial) order $S_{i j}$. Note the full $\Pi$ rotation over a period.

- the amplitude of the scattered light depends linearly on the applied voltage $V$;

- the scattered intensity is modulated at the frequency $\omega / 2 \Pi$ of the applied voltage;

- there is no dip in the scattering cross section when the $z$ optical wavevector transfer is zero. This is a proof that not only $\delta \varepsilon_{x z}$ and $\delta \varepsilon_{z x}$ contribute to the scattering, but also $\delta \varepsilon_{x x}$ and $\delta \varepsilon_{z z}\left(\varepsilon_{i j}\right.$ dielectric permittivity tensor at optical frequencies).

Once all these characteristic features are checked, one can deduce that flexoelectricity is indeed observed [13]. In fact, this proof is not complete : for instance, a thin nematic-like boundary layer at the glass-liquid interface could give rise to the same type of observations. Order of magnitude considerations [13] show however that the ratio of the scattered amplitudes $A^{\mathrm{bl}}$ (from nematic-like boundary layer flexoelectricity) over $A^{\mathrm{i}}$ (from bulk isotropic flexoelectricity) is of the order of a few $10^{-4}$ close to the transition, and should decrease with temperature. (A quick estimation of this ratio may be obtained if one remarks that there are only two lengths of importance in this problem, namely the boundary layer thickness $\xi$, and the electrode periodicity $\lambda_{e}$; thus $A^{\mathrm{bl}} / A^{\mathrm{i}} \simeq \xi / \lambda_{\mathrm{e}} \simeq 10^{-4}$ with $\lambda_{\mathrm{e}}=2 \times 10^{-2} \mathrm{~cm}^{-1}$ and $\left.\xi=2 \times 10^{-6} \mathrm{~cm}\right)$. Other linear effects such as charge 
injection may also be ruled out : a silicon dioxide layer protects the sample from injection and the only effect of free charges is the screening of the field at low frequencies (a few hertz or tens of hertz) [1] (in the absence of a protection layer, one does observe an enhancement of the scattered amplitude at low frequencies (a few hundred hertz), but this is suppressed by the $\mathrm{SiO}_{2}$ coating). Under these conditions it is then clear that one is dealing with flexoelectricity in the bulk of isotropic phases.

The detailed experimental set-up has been described previously $[4,13]$ : a light source $(\mathrm{HeNe} 124 \mathrm{~A}$ Spectra Physics or Argon 165 Spectra Physics Laser) irradiates the sample placed in the centre of a temperature controlled oven (10 mK stability, argon atmosphere). The scattered light is analysed at the frequency $\omega / 2 \Pi$ of the electrode potential, and in the direction corresponding to the desired wavevector conservation rule (EMI 9558A photomultiplier tube connected to a PAR 5202 or PAR 5206 Lock In amplifiers). The measurement of the scattered amplitude $A(\omega)$ at the frequency of the voltage, requires the use of a heterodyne optical detection scheme, which exhibits $10^{6}$ dynamic range both in amplitude and frequency. The determination of $A(\omega)$ is a direct measure of the orientational response function $\chi(\omega)$, apart from geometrical factors [13].

\section{Experimental results.}

As mentioned in the introduction we have studied two rod-like and two disk-like compounds. The octyl and nonylcyanobiphenyl have been purchased from British Drug Houses; traces of solvent were found and removed by pumping under vacuum, with the sample in its isotropic phase. They exhibit the following thermotropism :

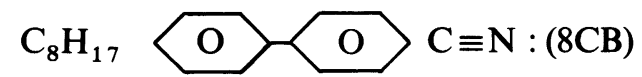

Crystal $\stackrel{21.5{ }^{\circ} \mathrm{C}}{\longrightarrow}$ Smectic A $\stackrel{33.50^{\circ} \mathrm{C}}{\longrightarrow}$ Nematic $\stackrel{41{ }^{\circ} \mathrm{C}}{\longrightarrow}$ Isotropic

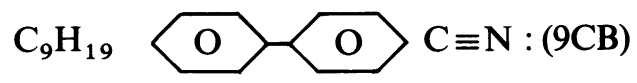

Crystal $\stackrel{42^{\circ} \mathrm{C}}{\longrightarrow}$ Smectic A $\stackrel{48{ }^{\circ} \mathrm{C}}{\longrightarrow}$ Nematic $\stackrel{50.2{ }^{\circ} \mathrm{C}}{\longrightarrow}$ Isotropic

The only sizeable difference between these two compounds is the range of existence of the nematic phase : $7.5^{\circ} \mathrm{C}$ for the octyl, $2.2^{\circ} \mathrm{C}$ for the nonyl. It is clear that smectic fluctuations are strong in the isotropic phase of the latter, and weaker in the former.

The hexaalkanoate truxene and hexaalkanoate triphenylene, both synthesized in our laboratory [5], have been purified by gel chromatography and freed from dust particles by successive filtering ( $\mathrm{G} 7$ millipore filters) and centrifugation.

The hexaalkanoate truxene is in fact a mixture characterized by the formula $\left(\mathrm{C}_{7,9,13} \mathrm{HATX}\right)$ :

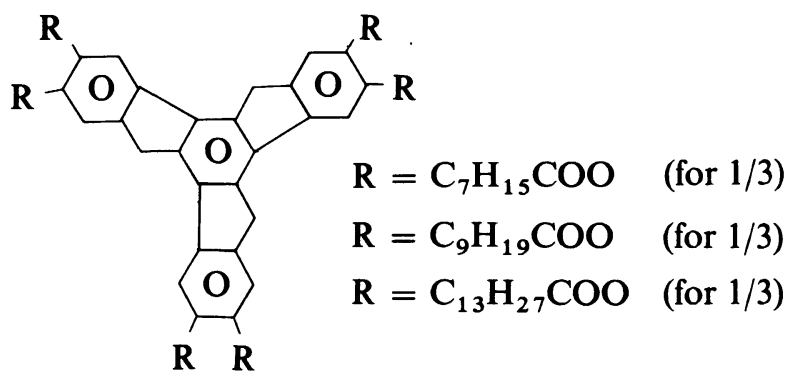

the successive phase transitions may be observed :

$$
\text { Crystal } \stackrel{55^{\circ} \mathrm{C}}{\longrightarrow} \text { Nematic } \stackrel{90{ }^{\circ} \mathrm{C}}{\longrightarrow} \text { Isotropic . }
$$

(Note the existence of a sizeable two-phase region $\left(2{ }^{\circ} \mathrm{C}\right)$ due to the mixture character of this compound.)

The hexaalkanoate triphenylene is a pure compound with formula $\left(\mathrm{C}_{11} \mathrm{HAT}\right)$ :

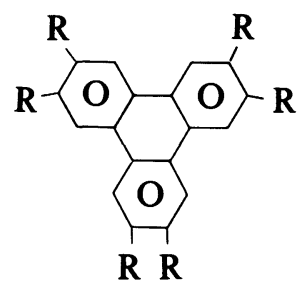

$$
\mathrm{R}=\mathrm{C}_{11} \mathrm{H}_{23} \mathrm{COO}
$$

and thermotropism :

$$
\mathrm{D}_{\mathrm{rd}} \stackrel{1111^{\circ} \mathrm{C}}{\longrightarrow} \mathrm{D}_{\mathrm{hd}} \stackrel{122^{\circ} \mathrm{C}}{\longrightarrow} \text { Isotropic . }
$$

This compound was chosen because of the weakness of the transition enthalpy ( $245 \mathrm{cal} . / \mathrm{mole})$, and of its good chemical stability (temperature shift of the order of $20 \mathrm{mK}$ per day, under argon atmosphere).

We have plotted in figures $2 a, b, c, d$ the typical frequency dependence of the flexoelectric response for each of the four compounds at different temperatures. All of them are found to be Lorentzian; the modulus of the scattered amplitude indeed obeys the simple law :

$$
|A(\omega)| \propto 1 / \sqrt{\omega^{2}+\omega_{\mathrm{r}}^{2}} .
$$

The typical relaxation frequencies of 8 and $9 \mathrm{CB}$ are of the order of those found for other rod-like liquid crystals. Those of the truxene hexaalkanoate are practically one order of magnitude smaller which suggests very large correlation domains or some kind of hindered rotation, if they are not due to the multicomponent character of this compound. This point should be checked with a pure sample. On the other hand, although the triphenylene hexaalkanoate corresponds to a fairly large molecule, it exhibits a relatively short correlation time, comparable to that of $9 \mathrm{CB}$ close to the transition temperature.

The temperature dependence of the scattered amplitudes $A\left(\omega \ll \omega_{\mathrm{r}}, T\right)$ are displayed in figures $3 a, b, c, d$ together with their inverse. The results concerning the 


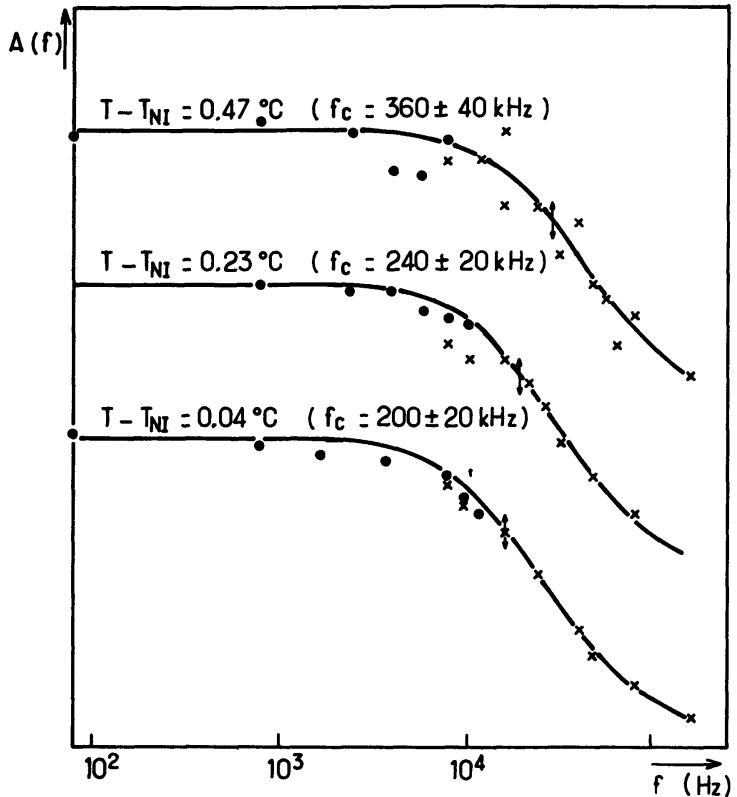

a)

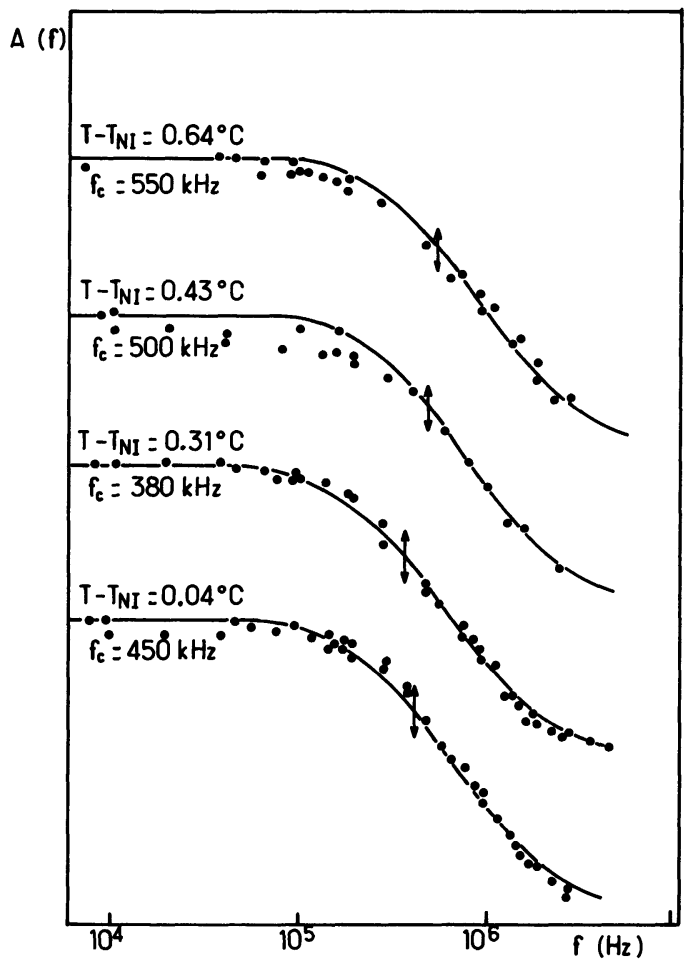

b)

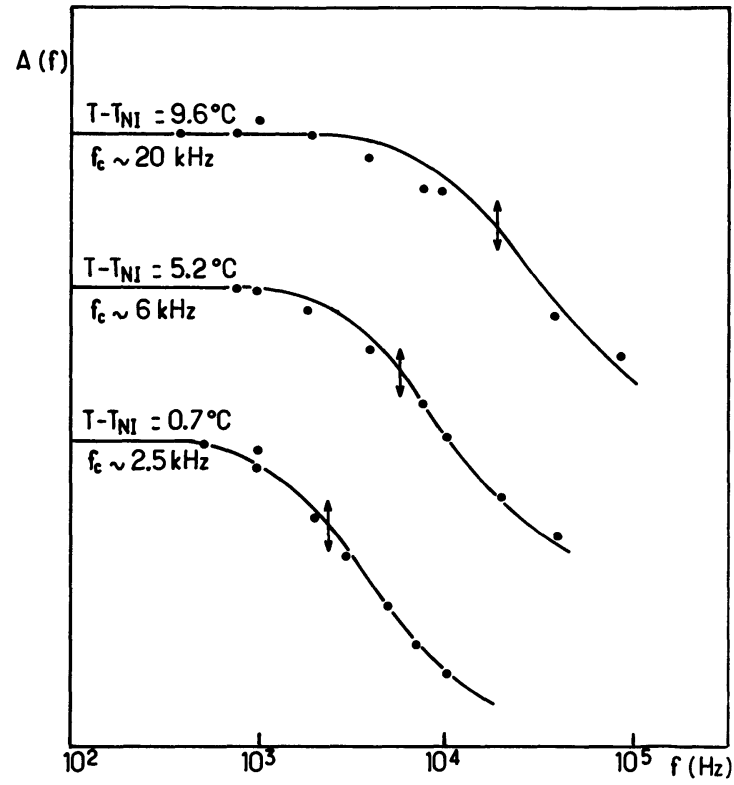

c)

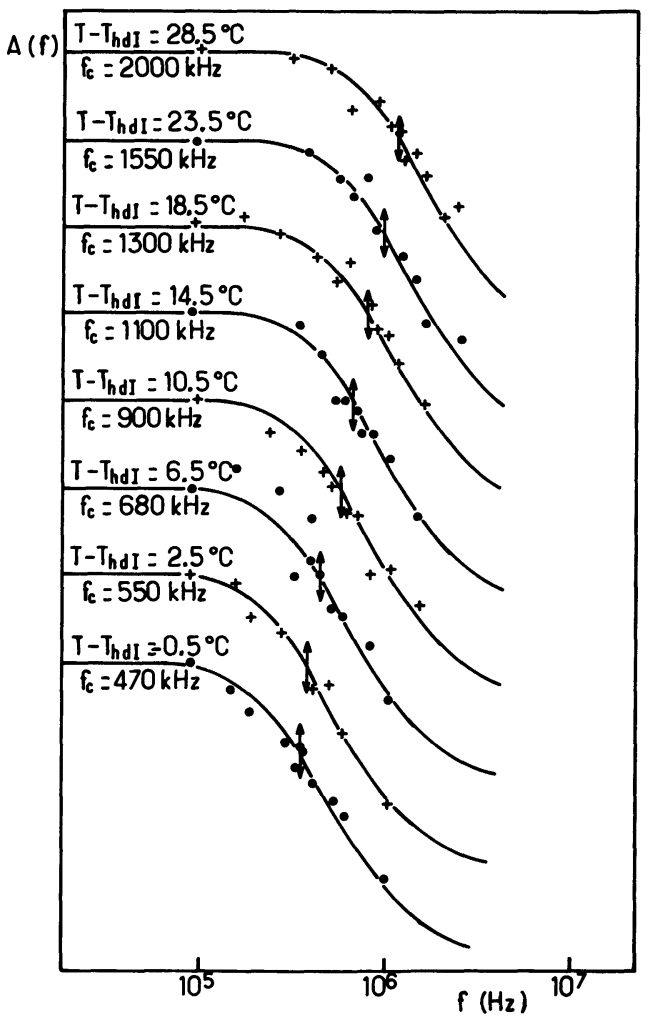

d)

Fig. 2. - Scattered amplitude $A(\omega)$ as a function of the frequency $f=\omega / 2 \Pi$, at different temperatures. The solid line is a fit according to a simple relaxation $\left(\omega^{2}+\omega_{\mathrm{r}}^{2}\right)^{-1 / 2}$. In all cases it provides a satisfactory account of the results. The low frequency plateau is proportional to the orientational response function $\chi$, as discussed in the text $\left(A_{\mathrm{N}}^{-1}\right.$ in the absence of smectic coupling, in the same limit $\omega_{\mathrm{r}}$ is proportional to $\left.A_{\mathrm{N}}\right)$. For clarity, the curves have been arbitrarily shifted along the ordinate axis. a) $8 \mathrm{CB}$ : note the monotonic decrease of $f_{\mathrm{c}}=\omega_{\mathrm{r}} / 2 \Pi$, while approaching the nematic phase. b) $9 \mathrm{CB}:$ the roll off frequency goes through a minimum value of $380 \mathrm{kHz}$ before reaching the nematic phase. This minimum correlates well with the maximum of the scattered amplitude $A(0), c) \mathrm{C}_{7,9,13} \mathrm{HATX}$ : the typical roll off frequencies are one order of magnitude smaller than in the $8 \mathrm{CB}$ and $9 \mathrm{CB}$ cases, but vary monotonically as in the $8 \mathrm{CB}$ case. $d$ ) $\mathrm{C}_{11} \mathrm{HAT}$ : the roll off frequency again behaves monotonically, and is of the same order of magnitude as the $8 \mathrm{CB}$ and $9 \mathrm{CB}$ ones. Note that because of the first-order character of the $D_{h d}-I$ transition, supercooled regions are accessible to experiment $\left(T_{D_{h d l}}=\right.$ transition temperature obtained with increasing temperature). 


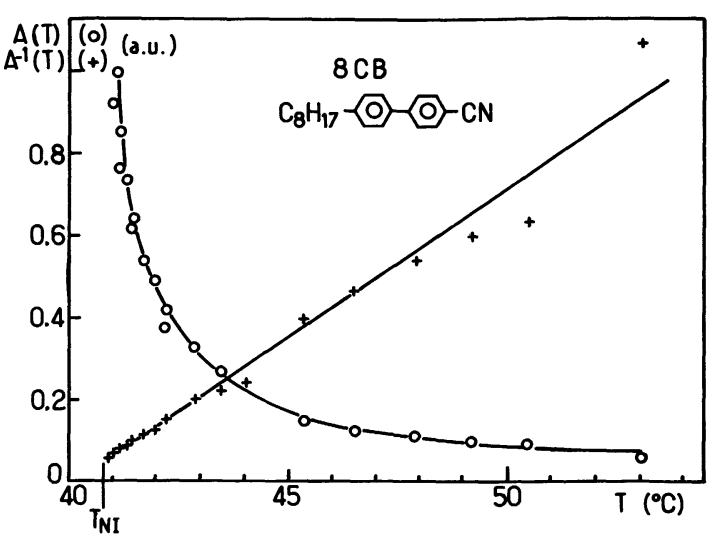

a)

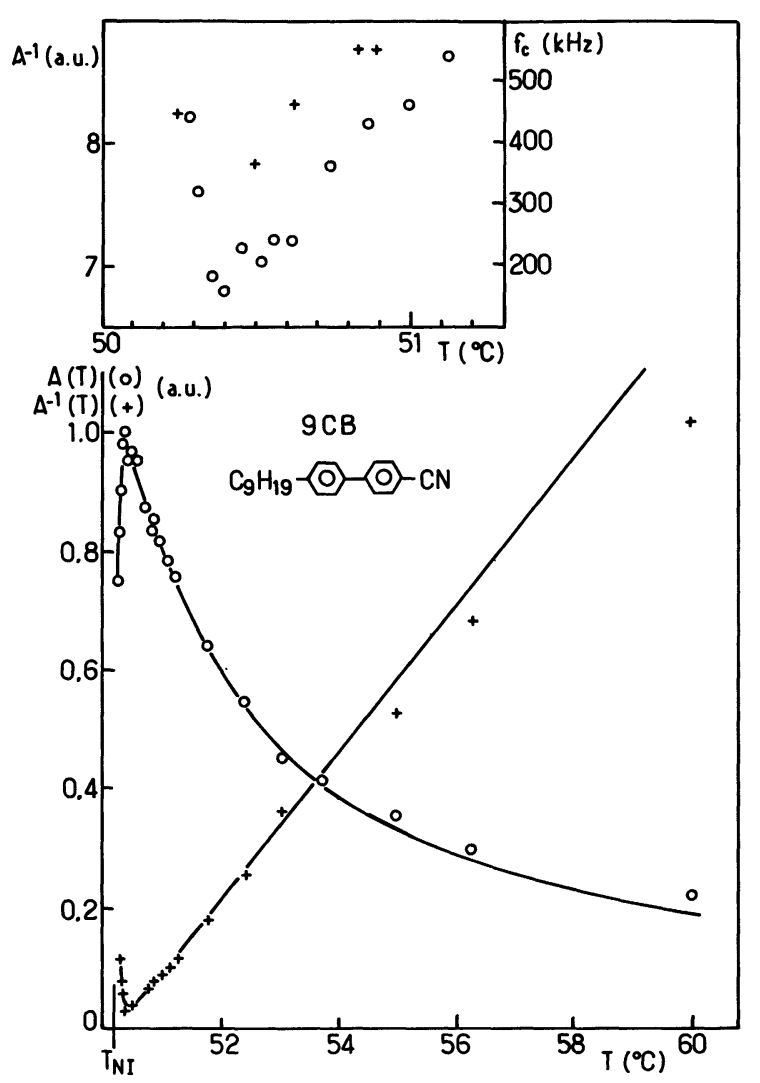

b)

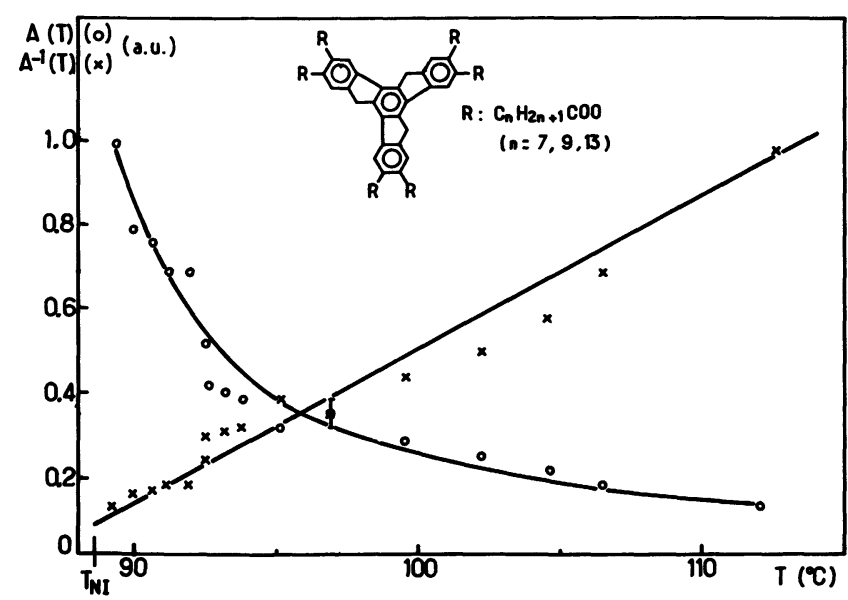

c)
8CB and the truxene hexaalkanoate agree with de Gennes theory of the nematic-isotropic transition [11, 12] : equation three, with $\gamma=1$ is obeyed reasonably well. On the other hand there is a striking deviation from simple power law behaviour close to the transition in the case of $9 \mathrm{CB}$; the minimum of the $A^{-1}(T)$ curve confirms earlier results obtained by Coles and Strazielle, on the same compound with a different technique $[14,15]$. The agreement of the two experiments shows that the anomalous behaviour cannot be blamed on experimental artifacts. It is also important to remark that the relaxation frequency $\omega_{r}$ reveals the same minimum as the inverse of the scattered amplitude (Fig. $3 b$ ). In the case of the direct isotropic-columnar phase transition, there is no reason a priori to expect de Gennes law to be valid : the orientational order parameter is not the relevant one at the $I-D_{h d}$ transition. It is thus not quite a

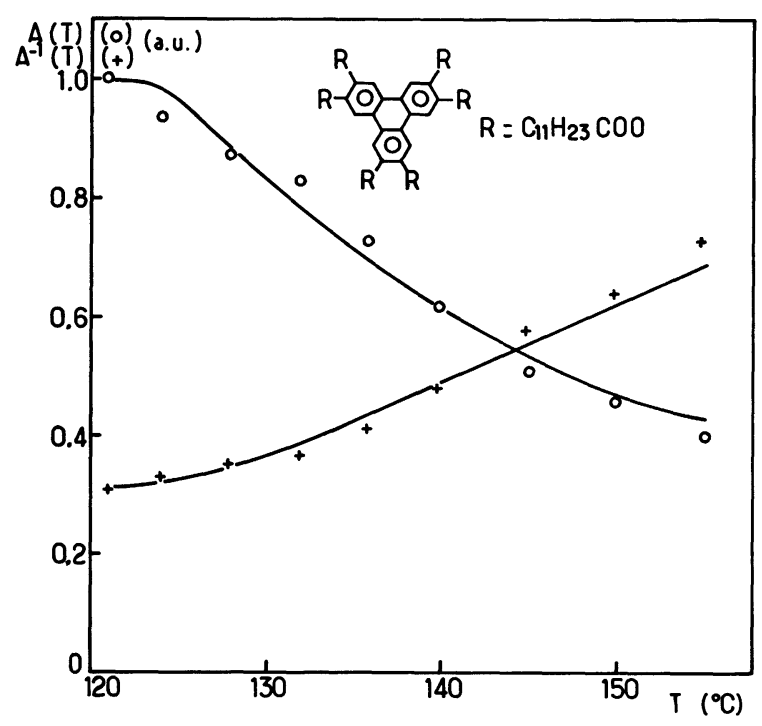

d)

Fig. 3. - Thermal dependence of the low frequency scattered amplitude ( $\circ$ ) and its inverse (+) : a) 8CB : note the linearity of the inverse scattered amplitude versus temperature. b) 9CB : the scattered amplitude clearly goes through a maximum $200 \mathrm{mK}$ away from the nematic-isotropic transition temperature. The linearity of the inverse scattered amplitude versus temperature is recovered far from the isotropic nematic transition. The solid line is a fit according to formula $15\left(t_{\mathrm{NI}}=T_{\mathrm{NI}}-T_{\rho} \simeq 0.01{ }^{\circ} \mathrm{C}\right)\left(t_{0}=-1.68^{\circ} \mathrm{C}\right.$; $\left.\tilde{\alpha}=0.14 ; \tilde{K}=3 \times 10^{-4}\right)$. The inset shows the correspondence between the minimum of the inverse of the scattered amplitude, and that of the roll off frequency $f_{c}=\omega_{\mathrm{r}} / 2 \Pi$. c) $\mathrm{C}_{7,9,13}$ HATX : note again the linearity of the inverse scattered amplitude versus temperature. $d$ ) $\mathrm{C}_{11} \mathrm{HAT}$ : note a trend toward saturation of the scattered amplitude close to the columnar-isotropic transition. The solid line is again a fit according to formula $15\left(t_{\mathbf{I D}_{\mathbf{h d}}}=\right.$ $\left.T_{\mathrm{ID}_{\mathrm{hd}}}-T_{\rho}=6^{\circ} \mathrm{C}\right)\left(t_{0}=1.5 ; \tilde{\alpha}=35 ; \tilde{K}=15\right)$. 
surprise if the $A^{-1}(T)$ curve of the triphenylene hexaalkanoate is not linear in $T$. There is a saturation of the flexoelectric response in the last ten degrees close to the transition. Similarly the relaxation frequency is not linear in $T$; the $A^{-1}(T)$ and $\omega_{\mathrm{r}}(T)$ dependences are similar but not exactly proportional over the whole temperature range under study.

\section{Interpretation.}

The flexoelectric response may be calculated from the de Gennes theory [11], of the isotropic to nematic transition when no important correlation with positional order is to be considered. At the lowest order [11, 1], the free energy reads :

$$
F-F_{0}=\int_{V}\left(\frac{A_{\mathrm{N}}}{2} S_{i j}^{2}+\frac{L_{1}}{2}\left(\nabla_{j} S_{i j}\right)^{2}+\frac{L_{2}}{2}\left(\nabla_{k} S_{i j}\right)^{2}+f_{1} S_{i j} \nabla_{i} E_{j}\right) \mathrm{d} v .
$$

As usual :

$$
A_{\mathrm{N}}=a_{\mathrm{N}}\left(T-T^{*}\right)
$$

$a_{\mathrm{N}}, f_{1}, L_{1}, L_{2}$, are practically independent of temperature in the range of interest. Minimizing with respect to $S_{i j}$ gives (for a field variation scale much larger than the correlation length $\xi^{2}=L_{i} / A_{\mathrm{N}}$ ):

$$
\left\langle S_{i j}\right\rangle=\frac{f_{1}}{A_{\mathrm{N}}} \nabla_{j} E_{i}
$$

or $\chi=-f_{1} / A_{\mathrm{N}}$ and $\gamma=1$, typical mean field exponent. The agreement with our results concerning the 8CB, the truxene mixture, and this simple description confirms earlier studies of various pretransitional phenomena [12]. The interesting fact is that the shape of the molecule (rod or disk) does not influence the way the nematic phase is approached. This is indeed currently a widely accepted idea but experimental confirmations are always welcomed (note that other $\gamma$ values should not be ruled out, a priori, since there is no complete treatment of the statistical mechanics of the N-I transition $[16,17])$.

As pointed out in the preceding section, the results concerning $9 \mathrm{CB}$ and $\mathrm{C}_{11} \mathrm{HAT}$ are more puzzling. A unique power law cannot describe the experimental behaviour. Although, $9 \mathrm{CB}$ and $\mathrm{C}_{11} \mathrm{HAT}$ do not exhibit the same low temperature phases, they share in common the fact that density modulation fluctuations strongly influence the orientational ones.

The extra free energy resulting from the orientation-translation coupling does not formally depend on the type of phase expected at low temperature (smectic, columnar, crystal) $[8,9]$ :

$$
F_{\mathrm{N} \rho}=\int_{V}\left\{\Gamma S_{i j}\left(\nabla_{i} \rho \nabla_{j} \rho-\frac{(\nabla \rho)^{2}}{3} \delta_{i j}\right)+\frac{K}{2} \rho^{2} S_{i j}^{2}\right\} \mathrm{d} v
$$

It has to be supplemented by the purely translational term [18] :

$$
F_{\rho}=\int_{V}\left\{\frac{A_{\rho}}{2} \rho^{2}+\frac{C}{2}\left(\left(\Delta+k_{0}^{2}\right) \rho\right)^{2}+\frac{D}{3} \rho^{3}+\frac{B}{4} \rho^{4}\right\} \mathrm{d} v .
$$

When density fluctuations take over, it is legitimate to minimize with respect to $S_{i j}$ (or to a Gaussian approximation of $S_{i j}$ ) :

$$
\left(A_{\mathrm{N}}+K \rho^{2}\right) S_{i j}=-\left\{\Gamma\left(\nabla_{i} \rho \nabla_{j} \rho-\frac{(\nabla \rho)^{2}}{3} \delta_{i j}\right)+f_{1} \nabla_{j} E_{i}\right\}
$$

hence :

$$
\left\langle\left(\left(A_{\mathrm{N}}+K \rho^{2}\right) S_{i j}\right)\right\rangle_{\nabla E}=-\left\{\left\langle\Gamma\left(\nabla_{i} \rho \nabla_{j} \rho-\frac{(\nabla \rho)^{2}}{3} \delta_{i j}\right)\right\rangle_{\nabla E}+f_{1} \nabla_{j} E_{i}\right\}
$$

The thermal average is understood to be taken in the presence of the field gradient. Or in a decoupling approximation :

$$
\left(A_{\mathrm{N}}+K\left\langle\rho^{2}\right\rangle\right)\left\langle S_{i j}\right\rangle_{\nabla E}=-\left\{\left\langle\Gamma\left(\nabla_{i} \rho \nabla_{j} \rho-\frac{(\nabla \rho)^{2}}{3} \delta_{i j}\right)\right\rangle_{\nabla E}+f_{1} \nabla_{j} E_{i}\right\}
$$


From linear response theory one knows that [19] :

$$
\left\langle\left(\nabla_{i} \rho \nabla_{j} \rho-\frac{(\nabla \rho)^{2}}{3} \delta_{i j}\right)(r)\right\rangle_{\nabla E}=-\beta f_{1} \int \mathrm{d} r^{\prime}\left\langle\left(\nabla_{i} \rho \nabla_{j} \rho-\frac{(\nabla \rho)^{2}}{3} \delta_{i j}\right)(\mathbf{r}) S_{k l}\left(\mathbf{r}^{\prime}\right)\right\rangle_{0} \nabla_{l} E_{k}\left(\mathbf{r}^{\prime}\right)
$$

(The thermal average on the right-hand-side is taken at zero field; $\beta=1 / k T$ ).

Making use of (8) (in zero field), eventually yields :

$$
\begin{array}{r}
\left\langle S_{i j}(\mathbf{r})\right\rangle_{\nabla E} \simeq \int \mathrm{d} r^{\prime}\left\{\Gamma^{2} \beta\left\langle\frac{\left(\nabla_{i} \rho \nabla_{j} \rho-\frac{(\nabla \rho)^{2}}{3} \delta_{i j}\right)(\mathbf{r})\left(\nabla_{k} \rho \nabla_{l} \rho-\frac{(\nabla \rho)^{2}}{3} \delta_{k l}\right)\left(\mathbf{r}^{\prime}\right)}{\left(A_{\mathrm{N}}+K\left\langle\rho^{2}\right\rangle\right)\left(A_{\mathrm{N}}+K \rho^{2}\left(r^{\prime}\right)\right)}\right\rangle_{0}+\right. \\
\left.+\frac{\delta_{\mathbf{r}-\mathbf{r}^{\prime}} \delta_{i k} \delta_{j l}}{A_{\mathrm{N}}+K\left\langle\rho^{2}\right\rangle}\right\} f_{1} \nabla_{l} E_{k}\left(\mathbf{r}^{\prime}\right) .
\end{array}
$$

Or for fields varying over distances much larger than the correlation lengths in the system :

$$
S_{i j}(\mathbf{r}) \simeq \chi_{i j k l} f_{1} \nabla_{l} E_{k}(r)
$$

with :

$$
\chi_{i j k l}=\int \mathrm{d} r \Gamma^{2} \beta\left\langle\frac{\left(\nabla_{i} \rho \nabla_{j} \rho-\frac{(\nabla \rho)^{2}}{3} \delta_{i j}\right)(0)\left(\nabla_{k} \rho \nabla_{l} \rho-\frac{(\nabla \rho)^{2}}{3} \delta_{k l}\right)(r)}{\left(A_{\mathrm{N}}+K\left\langle\rho^{2}\right\rangle\right)\left(A_{\mathrm{N}}+K \rho^{2}(r)\right)}\right\rangle_{\substack{0 \\+\delta_{i k} \delta_{j l} /\left(A_{\mathrm{N}}+K\left\langle\rho^{2}\right\rangle\right) .}}^{+}
$$

This formula appears as a generalization of relation (13) of [8]. Although strictly speaking, it is only valid when density fluctuations dominate, it also gives sensible results when only nematic ones are present. We thus believe that it may be used at least qualitatively to discuss the experimental results; the orientational response function appears as the sum of two terms. The first one $\left(\chi^{1}\right)$ is due to the short-range translational structure. In the limit $A_{\mathrm{N}}>K\left\langle l^{2}\right\rangle$, it has been shown to grow like $\xi_{\rho}^{3}\left(\xi_{\rho}\right.$ density modulation correlation function) [8]. In the opposite case, it essentially describes the phase correlation of the modulation. Further decoupling yields :

$$
\chi^{1} \sim \frac{\sum_{k}\left\langle k^{4} \rho^{2}(k) \rho^{2}(-k)\right\rangle}{\left(A_{\mathrm{N}}+K \sum_{k}\langle\rho(k) \rho(-k)\rangle\right)^{2}} \sim \frac{\xi_{\rho}^{3}}{\left(T-T^{*}+\alpha \xi_{\rho}\right)^{2}} .
$$

(For the evaluations of the integrals see [8];

$$
\alpha=k_{\mathrm{B}} T K / 8 \Pi C a_{n}
$$

in the harmonic approximation $\left.\xi_{\rho}^{2}=4 k_{0}^{2} C / A_{\rho}\right)$.

Depending on the value of the ratio $\left(T^{*}-T_{\rho}\right) a_{\rho}^{1 / 3} /\left(\alpha 2 k_{0}\right)^{2 / 3} C^{1 / 3}\left(A_{\rho}=a_{\rho}\left(T-T_{\rho}\right)\right)$, the curve $\chi^{1}(T)$ may be either monotonically decreasing with $T$, or exhibit an $S$ shape.

The second term $\chi^{2}$ is more classical; in the regime $\left.A_{\mathrm{N}}\right\rangle K\left\langle\rho^{2}\right\rangle$, it gives the standard response whereas in the opposite case its contribution vanishes when $\xi_{\rho} \rightarrow \infty$,

In the same approximation as (13):

$$
\chi^{2} \sim 1 /\left(T-T^{*}+\alpha \xi_{p}\right) .
$$

One thus easily understands that the total response function $\chi$, may be either monotonic, or show a kink. One must bear in mind that, since the isotropic-anisotropic phase transitions are first-order, one will usually not observe the last part of the curve. It thus seems possible to describe the experimental observations on the basis of formula 12. According to (13) and (14) :

$$
\chi \sim\left(\frac{\tilde{K t^{-3 v}}}{\left(t-t_{0}+\tilde{\alpha} t^{-v}\right)^{2}}+\frac{1}{t-t_{0}+\tilde{\alpha} t^{-v}}\right)
$$

$\left(t=T-T_{\rho} ; t_{0}=T^{*}-T_{\rho} ; \tilde{\alpha}=\left(2 k_{0} \alpha\right) C^{1 / 2} / a_{\rho}^{1 / 2} ; \tilde{K}=\Gamma^{2} k_{\mathrm{B}} T k_{0}^{5} / 36 \Pi C^{1 / 2} a_{\mathrm{N}} a_{\rho}^{3 / 2} ;\right.$ in the harmonic approximation $v=1 / 2$ ). 
The fit of the results concerning the $9 \mathrm{CB}$ and the $\mathrm{C}_{11}$ HAT according to formula 15 is displayed in figures $3 b$ and $d$. An agreement compatible with the experimental accuracy is obtained over a rather large range of parameters $\tilde{\alpha}$ and $\tilde{K}$. On the other hand the temperatures $T^{*}$ and $T_{\rho}$ are always found to be within a few degrees of the actual transition temperature. We take this result as an indication that our interpretation points in the right direction since any simple power law fit gives meaningless values of $T^{*}$ in the HAT case, and of course no maximum is observed without translational fluctuations. For both $9 \mathrm{CB}$ and HAT the $\chi^{2}$ contribution is found to be more important than $\chi^{1}[20]$.

Recent experiments [21] seem to blame the existence of a maximum in the Rayleigh scattering intensity versus temperature in the $9 \mathrm{CB}$ on the lack of purity of the compounds. From our experiment it is very clear that it cannot be a trivial effect such as those connected to two-phase regions. On the other hand slight changes in the $T^{*}-T_{\rho}$ values do influence in an essential way the existence of the maximum. Furthermore impurity concentrations can be coupled to $S$, via an $S^{2} C^{2}$ term, but the corresponding fluctuations have no reason to go critical.

In principle the dynamical behaviours of $\chi^{1}$ and $\chi^{2}$ have no reason to be equivalent, and the resulting $\chi(\omega)$ should not be Lorentzian. $\chi^{1}$ is governed by the density modulation correlation time (and in a non essential way it is different from a Lorentzian shape [22]), and $\chi^{2}$ by the angular correlation time. In practice however, we believe that it is hard to demonstrate clearly the difference between Lorentzian and non Lorentzian shapes.

- Furthermore $\chi^{-1}(T)$ and $\omega_{\mathrm{r}}(T)$ should have different temperature dependences, because of the $\chi^{1}$ contribution. The predominance of the $\chi^{2}$ term however explains why the experimental $\chi^{-1}(T)$ and $\omega_{\mathrm{r}}(T)$ correspond fairly well for both $9 \mathrm{CB}$ and HAT. In particular a minimum of $\omega_{\mathrm{r}}(T)$ corresponds to the maximum of $A(T)$ in the case of $9 \mathrm{CB}$. The slight discrepancy of the $\omega_{\mathrm{r}}(T)$ and $A^{-1}(T)$ curves close to the transition temperature in the case of HAT might be an indication of the existence of the $\chi^{1}$ contribution.

Finally we note that at this level of approximation the translational fluctuations play the same rôle whether they are of smectic, columnar or crystalline type. Although the sign and magnitude of $\Gamma$ changes from one case to the other only its square enters formula 12 .

\section{References}

[1] Marcerou, J. P., Prost, J., Phys. Lett. 66A (1978) 218.

[2] Prost, J., Marcerou, J. P., J. Physique 38 (1976) 315.

[3] In fact, dipolar contributions to flexoelectricity of the type originally introduced by MEYER (Phys. Rev. Lett. 22 (1969) 918), might exist in isotropic phases in the form introduced by STRaley (Phys. Rev. A 14 (1976) 1835). Their experimental relevance has been discussed in (1), see also :

Marcerou, J. P., Prost, J., Mol. Cryst. Liq. Cryst. 58 (1980) 259. Conformational flexoelectrocity has also been introduced recently by G. DURAND (Journées Françaises - Cristaux Liquides Alpes d'Huez (1983)). Note that (5) does not imply any microscopic assumption on the nature of the coupling; terms such as $\nabla_{i} S_{i j} E_{j}$ are equivalent to $S_{i j} \nabla_{i} E_{j}$ provided an integration by parts is performed.

[4] Prost, J., Pershan, P. S., J. Appl. Phys. 47 (1976) 2298. Details may be found in : MARCERoU, J. P., Thèse de spécialité, Bordeaux N 1412 (1978).

[5] We are greatly indebted to NGUYEN HUU TINH, C. DESTRADE and P. FoUCHER for providing us with the discoid compounds.

[6] Foucher, P., Thèse de spécialité, No Ordre 1839, Bordeaux (1983).

[7] Levelut, A. M., Proceedings Int. Conf. Liq. Cryst. Bangalore (Heyden and Son) (1980) 21.

Destrade, C., Nguyen HuU Tinh, Gasparoux, H., Malthete, J., Levelut, A. M., Mol. Cryst. Liq. Cryst. (1981) 71.
[8] Gohin, A., Destrade, C., Gasparoux, H., Prost, J., J. Physique 44 (1983) 427.

A somewhat similar approach has been developed independently : ANISOMOV, M. A., GoRODETSKY, E. E., Podneks, V. E., J.E.T.P. Lett. 8 (1983) 37.

[9] DebYe, P., Phys. Z. 22 (1921) 63.

[10] Buckingham, A. D., J. Chem. Phys. 30 (1959) 1580.

[11] De Gennes, P. G., Mol. Cryst. Liq. Cryst. 12 (1971) 193.

[12] De Gennes, P. G., The Physics of Liquid Crystals (Clarendon, Oxford) 1974.

[13] More details can be found in : MARCERou, J. P., Thèse de doctorat d'Etat, Bordeaux N 734 (1982).

[14] Coles, H. J., Strazielles, C., Mol. Cryst. Liq. Cryst. 55 (1979) 237.

[15] Pouligny, B., Marcerou, J. P., Lalanne, J. R., Coles, H. J., Mol. Phys. 49 (1983) 583.

[16] Lin LeI, Phys. Rev. Lett. 43 (1979) 1604.

[17] Priest, R. G., Lubensky, T. C., Phys. Rev. B 13 (1976) 4159.

[18] Prost, J., Barois, P., J. Chem. Phys. 80 (1983) 65.

[19] Forster, D., in Hydrodynamic fluctuations, Broken Symmetry and Correlation functions. Frontier in Physics (Benjamin) 1975.

[20] Note that in the case of the p-n-heptyloxybenzylidene$\mathrm{p}^{\prime}$-n-butylaniline the $\tilde{K}$ term was found to be larger than the $\tilde{\alpha}$ one (8).

[21] DE JEU, W., private communication.

Zhang Shu-Lin, Peng Zheng-Yu, Wu Jin, Shen Tie-Han and Wu Nai-QIang, Mol. Cryst. Liq. Cryst. 91 (1983) 295.

[22] Prost, J., calculations underway. 